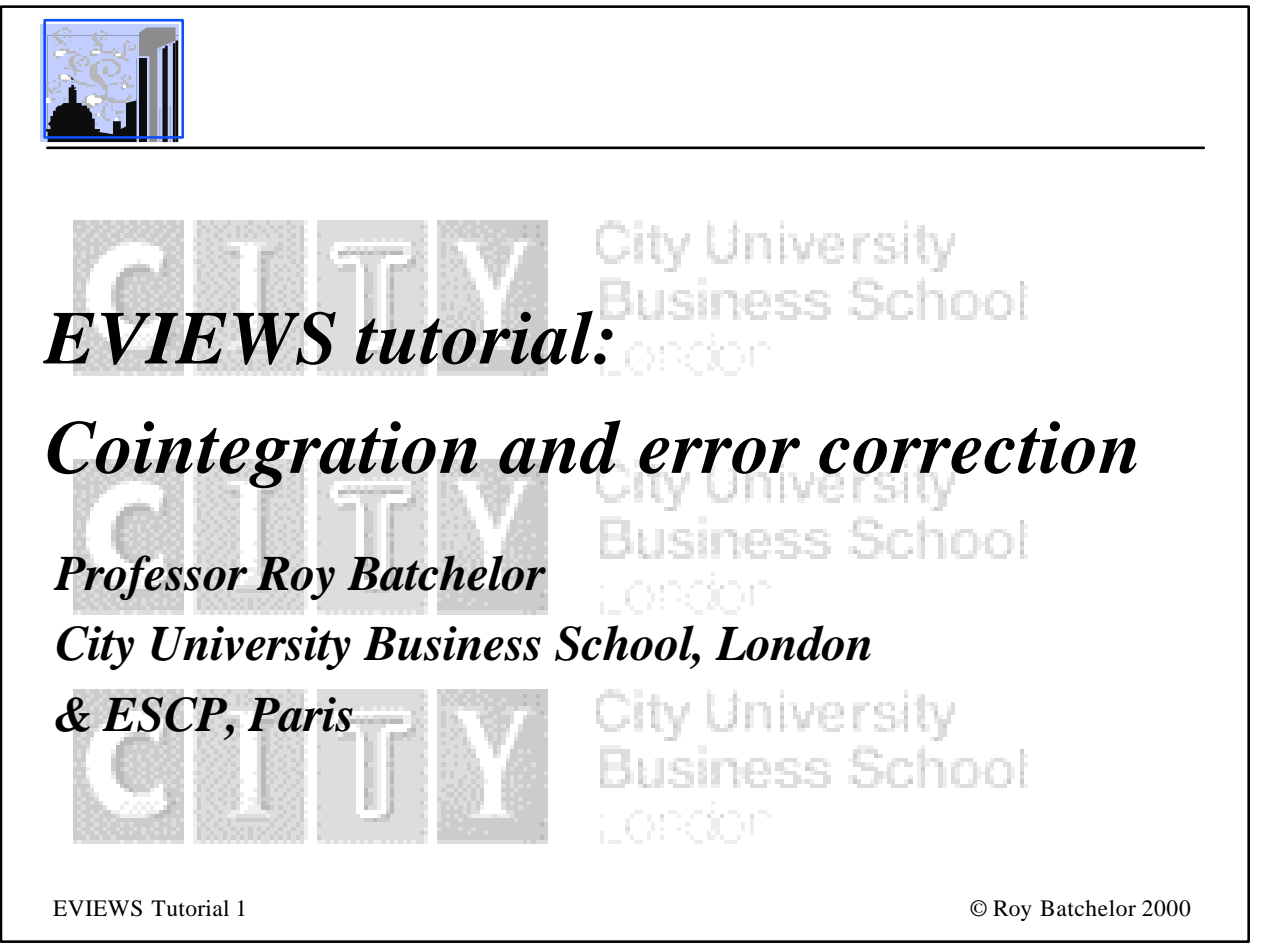

\title{
EVIEWS
}

$\square$ On the City University system, EVIEWS 3.1 is in

Start/ Programs/Departmental Software/CUBS

$\square$ Analysing stationarity in a single variable using VIEW

$\square$ Analysing cointegration among a group of variables

$\square$ Estimating an ECM model

$\square$ Estimating a VAR-ECM model 


\section{The FT500M workfile}

\begin{tabular}{|c|c|c|c|c|c|c|}
\hline \multicolumn{7}{|l|}{ 매매 EViews } \\
\hline File Edit $\underline{\text { Qbjects }}$ & View & Quick & ns $\underline{\text { Window }}$ & elp & & \\
\hline \multicolumn{7}{|c|}{$\square$ Workfile: UNTITLED $\quad-|\square| x \mid$} \\
\hline \multicolumn{7}{|c|}{ View/Procs/Objects S Save/Label+l-1 Show/Fetch/Store /Delete/Genr/Sample } \\
\hline \multicolumn{5}{|c|}{$\begin{array}{lrll}\text { Range: } & 1975: 01 \text { 2000:12 } & \text { Filter: }{ }^{*} & \text { Default Eq: None } \\
\text { Sample: } 1975: 01 \text { 2000:12 } & & \\
\end{array}$} & & \\
\hline \multicolumn{7}{|c|}{ 回 c } \\
\hline \multirow{4}{*}{\multicolumn{7}{|c|}{ 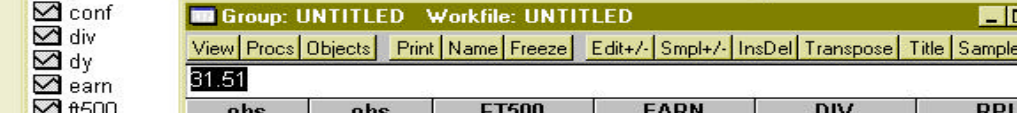 }} \\
\hline & & & & & & \\
\hline & & & & & & \\
\hline & & obs & FT500 & EARN & DIV & RPI \\
\hline \multirow{8}{*}{$\begin{array}{l}\Delta_{\text {infl }}^{\text {growth }} \\
\Delta_{\text {prod }}^{\text {resid }} \\
\Delta_{\text {return }}^{\text {ri }} \\
\Delta_{\text {tb3 }}\end{array}$} & 1999:08 & 1999:08 & 14709.42 & 495.4300 & 326.5500 & $166.2 \mathrm{C}$ \\
\hline & 1999:09 & 1999:09 & 14178.95 & 505.1300 & 327.5300 & 166.5[ \\
\hline & 1999:10 & 1999:10 & 14572.28 & 501.9700 & 327.8800 & $166.7 \mathrm{C}$ \\
\hline & 1999:11 & 1999:11 & 15725.26 & 520.3600 & 333.3800 & 167.3[ \\
\hline & 1999:12 & $1999: 12$ & 16694.16 & 515.4100 & 330.5400 & $166.6 \mathrm{C}$ \\
\hline & 2000:01 & 2000:01 & 15612.37 & 518.5100 & 329.4200 & 167.5[ \\
\hline & 2000:02 & 2000:02 & 15826.98 & 491.8300 & 313.3700 & 168.4[ \\
\hline & 2000:03 & 2000:03 & 16355.85 & 521.3900 & 310.7600 & $170.1 \mathrm{C}$ \\
\hline \multirow[t]{7}{*}{+2} & 2000:04 & $2000: 04$ & 15784.34 & 528.4300 & 310.9500 & $170.7 \mathrm{C}$ \\
\hline & 2000:05 & $2000: 05$ & 15658.66 & 564.4800 & 311.6100 & 171.1[ \\
\hline & 2000:06 & $2000: 06$ & 15769.05 & 544.1400 & 309.0700 & 170.5[ \\
\hline & 2000:07 & $2000: 07$ & 15907.72 & 531.5000 & 305.4300 & 170.5[ \\
\hline & 2000:08 & $2000: 08$ & 16525.57 & 556.6000 & 309.0300 & $171.7 \mathrm{C}$ \\
\hline & 2000:09 & $2000: 09$ & NA & NA & NA & NA \\
\hline & mกnก.1ก & วกกก-1ก & NAA & NA & MA & (C) Roy Batc \\
\hline
\end{tabular}

\section{Data transformation}

$\square$ Generate a series for the natural log of the FT500 index (lft500)

Test for stationarity in

- the level of this series

- the first difference of this series (dlft500)

$\square$ Results show that lft500 is an I(1) variable
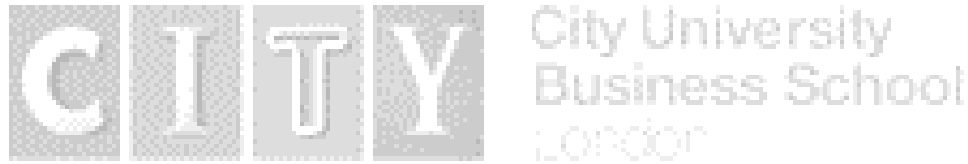


\section{Generate $\ln ($ FT500)}

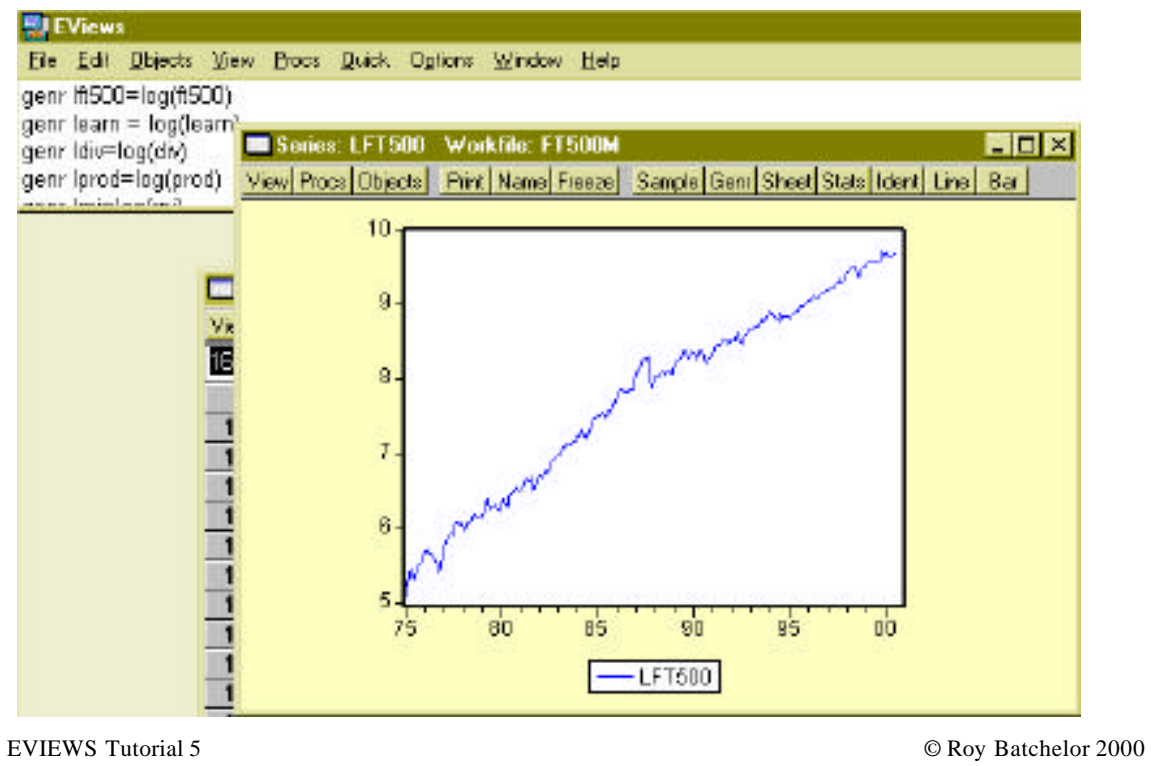

$\square$ Series: LFT500 Workfile: FT500M $-|\square| x \mid$

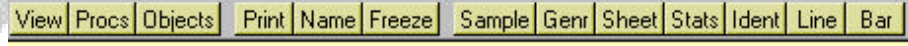

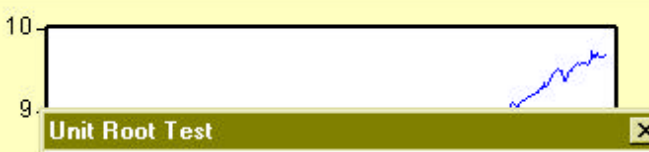

8. Test Type: Include in test equation:

- Augmented Dickey-Fuller + Intercept

$7 . \quad$ Phillips-Perron

Irend and intercept

Test for unit root in: $\quad$ None

6. Level

1st difference

2nd difference

原

5.

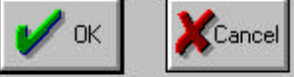




\begin{tabular}{|c|c|c|c|c|c|}
\hline \multicolumn{6}{|c|}{ ADF results: level } \\
\hline \multicolumn{5}{|c|}{ 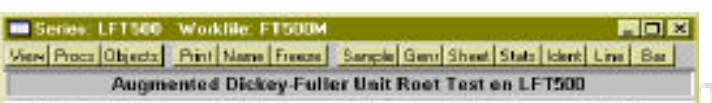 } & \multirow{2}{*}{$\begin{array}{l}\text { wersily } \\
\text { sohool }\end{array}$} \\
\hline ADF Test St:aistic & ${ }^{1.437515}$ & 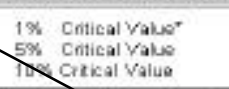 & $\begin{array}{l}-3.4537 \\
-2.27712 \\
-2.5719\end{array}$ & & \\
\hline \multicolumn{5}{|c|}{ TMackmonn crtical walues ber rejection of hyponthy of a unt root. } & \\
\hline \multicolumn{4}{|c|}{ 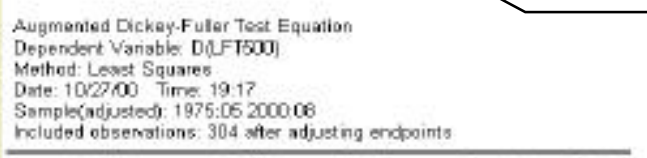 } & & $\begin{array}{l}\text { The hypothesis that } \\
\text { lft500 has a unit root } \\
\text { cannot be rejected }\end{array}$ \\
\hline Yariablo & Coefficient & Std Eror 1-Shastistic & Prob. & & \\
\hline 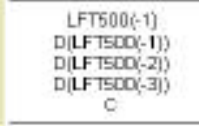 & 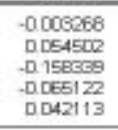 & 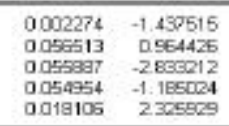 & $\begin{array}{l}0.1516 \\
0.3515 \\
0.0023 \\
0.2393 \\
0.0278\end{array}$ & & \\
\hline 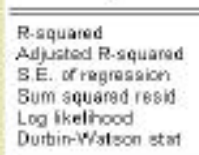 & 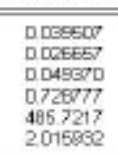 & 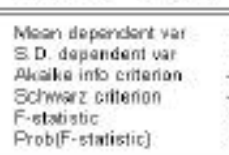 & 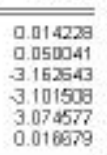 & & $\begin{array}{l}\text { ersty } \\
\text { school }\end{array}$ \\
\hline EVIEWS Tutorial 7 & & & & & ( ) Roy Batchelor 2000 \\
\hline
\end{tabular}

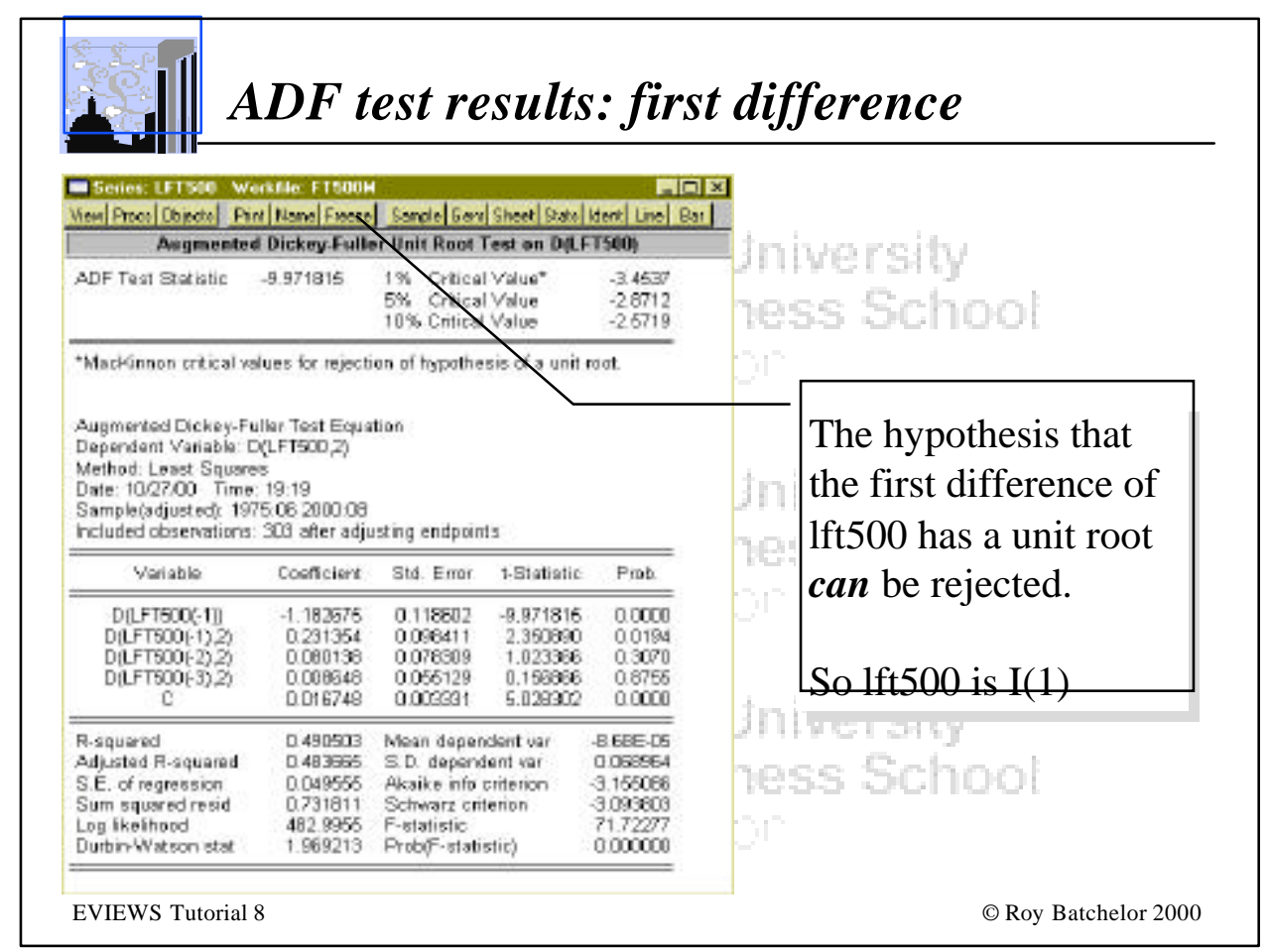




\section{Cointegration: two variables}

$\square$ The variables lft500 (log of stock index) and ldiv (log of dividends per share) are both I(1)

$\square$ We can test whether they are cointegrated

- that is, whether a linear function of these is $I(0)$

- An example of a linear function is

$$
1 \mathrm{ft} 500_{\mathrm{t}}=\mathrm{a}_{0}+\mathrm{a}_{1} \operatorname{ldiv}_{\mathrm{t}}+\mathrm{u}_{\mathrm{t}}
$$

when $u_{t}=\left[1 f t 500_{t}-a_{0}-a_{1} l d i v\right]$ might be $\mathrm{I}(0)$

$\square$ The expression in brackets [] is called the cointegrating vector, which has normalised coefficients $\left[1,-a_{0},-a_{1}\right]$

\section{Form new group ...}

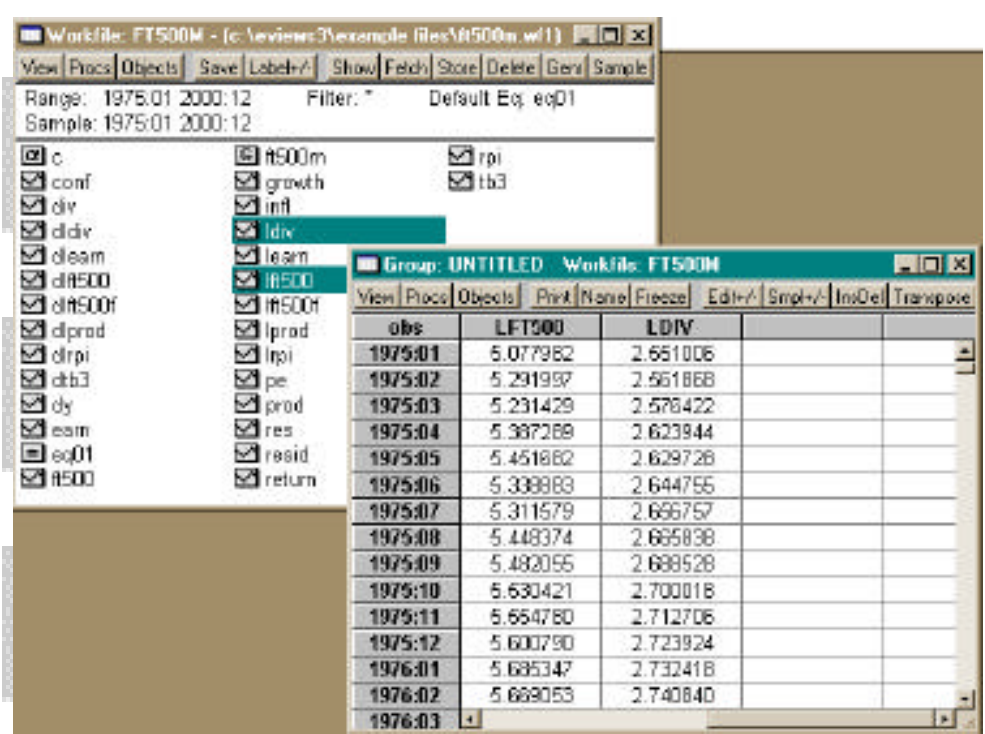




\section{Common trends?}

口Group: UNTITLED Workfile: FT500M $\quad-\mid \square \times$

View/Procs/Objects] Print/Name|Freezel Sample/Sheet/Stats/Spec]

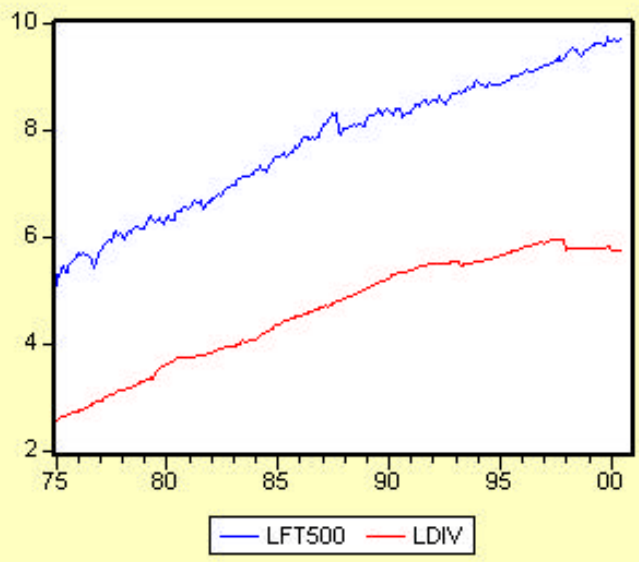

\section{Engle-Granger: first stage regression}

DEquation: UNTITLED Workfile: FT500M

\section{$-|\square| a \mid$}

View/Procs/Objects Print/Name/Freeze Estimate|Forecast/Stats/Resids

Dependent Variable: LFT500

Method: Least Squares

Date: 11/02/00 Time: 11:01

Sample: 1975:01 1995:12

Included observations: 252

\begin{tabular}{ccccc}
\hline \hline Variable & Coefficient & Std. Error & t-Statistic & Prob. \\
\hline \hline C & 2.388322 & 0.040888 & 58.41105 & 0.0000 \\
LDV & 1.149353 & 0.009165 & 125.4075 & 0.0000 \\
\hline
\end{tabular}

R-squared $\quad 0.984353$ Mean dependent var 7.395240

$\begin{array}{lll}\text { Adjusted R-squared } \quad 0.984290 & \text { S.D. depgldent var } 1.117301\end{array}$

S.E. of regression $\quad 0.140042$ Akaike info criterion -1.085842

Sum squared resid $\quad 4.902952$ Sh hwarz criterion $\quad-1.057830$

Log likelihood $\quad 138.8160$ F-statistic 15727.05

$\begin{array}{llll}\text { Durbin-Watson stat } \quad 0.167088 & \text { Prob(F-statistic) } \quad 0.000000\end{array}$ 


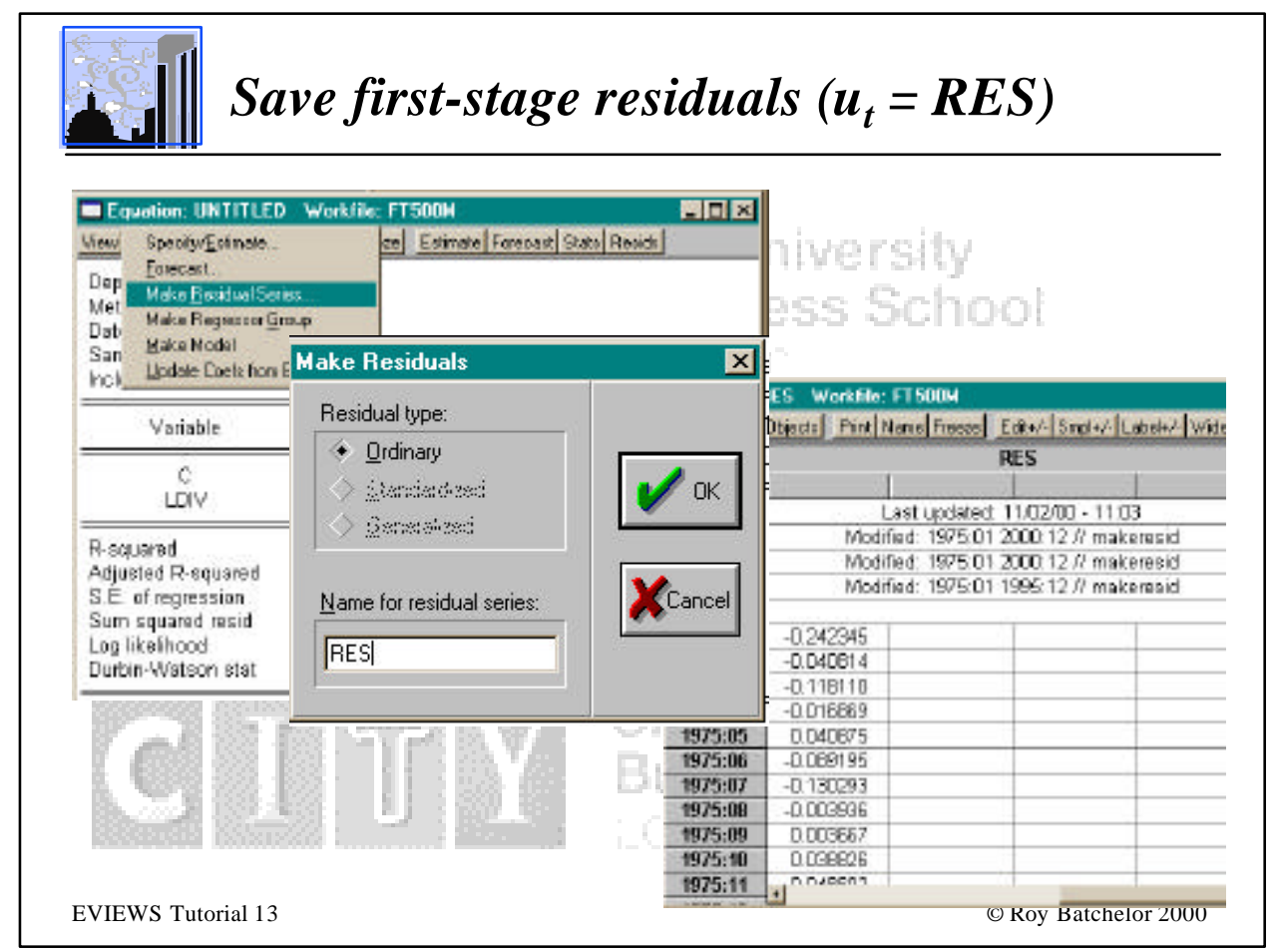

\begin{tabular}{|c|c|c|c|c|c|}
\hline \multicolumn{6}{|c|}{ Engle-Granger:stage two (ECM) regression } \\
\hline \multicolumn{3}{|c|}{$\square$ Equation: UNTITLED Workfile: FT500M } & & $-|\nabla x|$ & \multirow{5}{*}{ 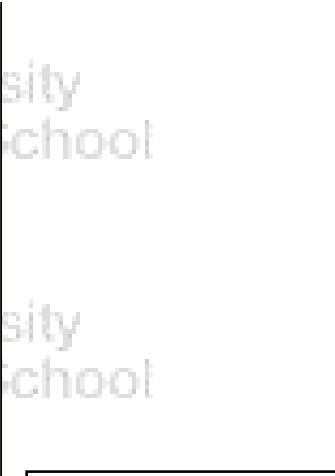 } \\
\hline \multicolumn{5}{|c|}{ 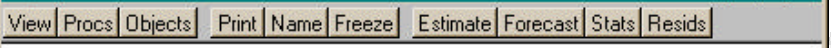 } & \\
\hline \multicolumn{5}{|c|}{$\begin{array}{l}\text { Dependent Variable: DLFT500 } \\
\text { Method: Least Squares } \\
\text { Date: } 11 / 02 / 00 \text { Time: } 11: 06 \\
\text { Sample(adjusted): } 1975: 03 \text { 1995:12 } \\
\text { Included observations: } 250 \text { after adjusting endpoints } \\
\end{array}$} & \\
\hline Variable & Coefficient & Std. Error & t-Statistic & Prob. & \\
\hline $\begin{array}{l}\text { C } \\
\text { DLFT5O0(-1) } \\
\text { DLDIV } \\
\text { DLDIV(-1) } \\
\text { RES(-1) }\end{array}$ & $\begin{array}{r}0.010568 \\
0.059286 \\
0.148933 \\
0.125376 \\
-0.073868\end{array}$ & $\begin{array}{r}0.005777 \\
0.062509 \\
0.257720 \\
0.255328 \\
0.025017\end{array}$ & $\begin{array}{r}1.829286 \\
0.948434 \\
0.577887 \\
0.491037 \\
-2.952702 \\
\end{array}$ & $\begin{array}{l}0.0686 \\
0.3438 \\
0.5639 \\
0.6238 \\
0.0035\end{array}$ & \\
\hline $\begin{array}{l}\text { R-squared } \\
\text { Adjusted R-squared } \\
\text { S.E. of regression } \\
\text { Sum squared resid } \\
\text { Log likelihood } \\
\text { Durbin-Watson stat }\end{array}$ & $\begin{array}{l}0.035776 \\
0.020034 \\
0.053443 \\
0.699762 \\
380.0748 \\
1.929673\end{array}$ & $\begin{array}{l}\text { Mean depe } \\
\text { S.D. depen } \\
\text { Akaike info } \\
\text { Schwarz cr } \\
\text { F-statistic } \\
\text { Prob(F-stat }\end{array}$ & $\begin{array}{l}\text { dent var } \\
\text { ent var } \\
\text { riterion } \\
\text { erion } \\
\text { tic) }\end{array}$ & $\begin{array}{r}0.014948 \\
0.053987 \\
-3.000598 \\
-2.930169 \\
2.272610 \\
0.062054\end{array}$ & $\begin{array}{l}\text { About } 7 \% \text { of } \\
\text { disequilibrium } \\
\text { "corrected" each }\end{array}$ \\
\hline EVIEWS Tutorial 14 & & & & & ( ) Roy Batchelor 2000 \\
\hline
\end{tabular}




\begin{tabular}{|c|c|c|c|c|c|}
\hline \multicolumn{6}{|c|}{ General model: stage one (I(1) variables) } \\
\hline \multirow{2}{*}{\multicolumn{5}{|c|}{ 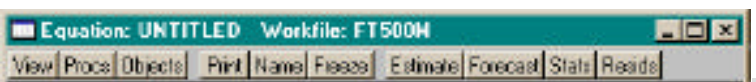 }} & \multirow{4}{*}{$\begin{array}{l}\text { rersity } \\
\text { schollow }\end{array}$} \\
\hline & & & & & \\
\hline \multicolumn{5}{|c|}{ 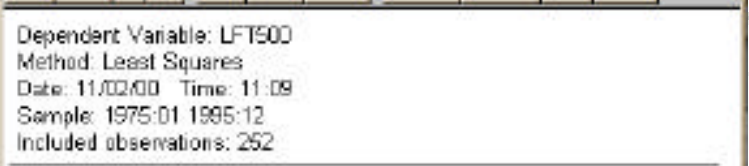 } & \\
\hline Yariabls & Coefficient & STd Emor & t-Statistic & Prob. & \\
\hline 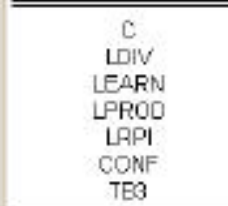 & $\begin{aligned} 4.438814 \\
0.824553 \\
0.400457 \\
-0.603047 \\
0.041973 \\
0.0575704 \\
-1.057279\end{aligned}$ & 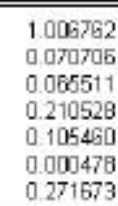 & 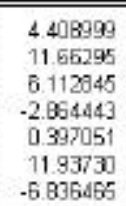 & $\begin{array}{l}0.0000 \\
0.0000 \\
0.0000 \\
0.0045 \\
0.6917 \\
0.00000 \\
0.00000\end{array}$ & $\begin{array}{l}\text { sershy } \\
\text { school }\end{array}$ \\
\hline 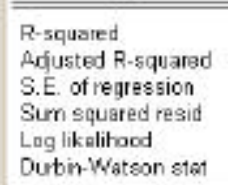 & $\begin{array}{l}0.993328 \\
0.993164 \\
0.092376 \\
2.090554 \\
246.2175 \\
0.451959\end{array}$ & \multicolumn{2}{|c|}{ 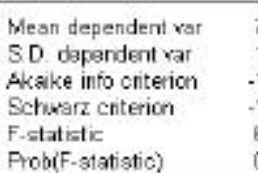 } & $\begin{array}{l}7.395240 \\
1.117301 \\
-1.895520 \\
-1.800490 \\
6079.104 \\
0.0000000\end{array}$ & $\begin{array}{l}\text { Jersthy } \\
\text { scholit }\end{array}$ \\
\hline EVIEWS Tutorial 15 & & & & & (- Roy Batchelor 2000 \\
\hline
\end{tabular}

\begin{tabular}{|c|c|c|c|c|c|}
\hline \multicolumn{6}{|c|}{ General model: stage two } \\
\hline \multicolumn{5}{|c|}{ 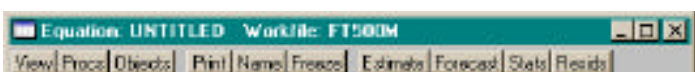 } & \multirow{4}{*}{ 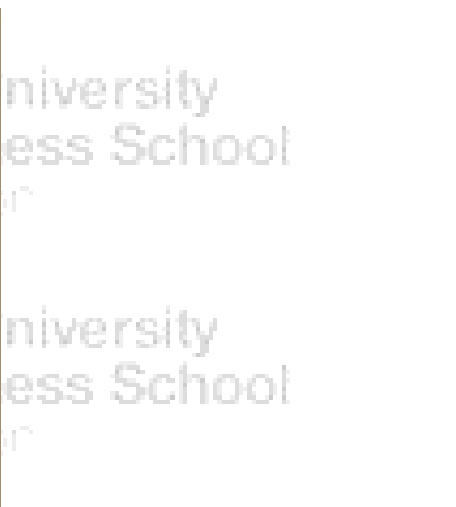 } \\
\hline \multicolumn{5}{|c|}{ 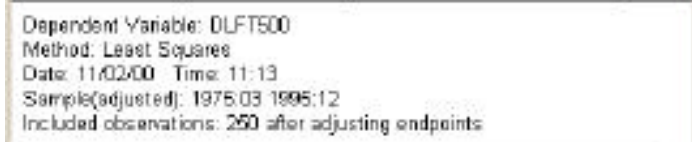 } & \\
\hline Variable & Coefficien: & Fitd Error & t-Statistic & Prob & \\
\hline 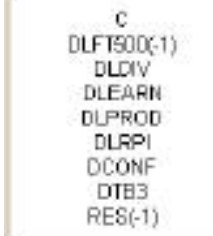 & 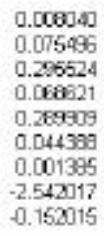 & 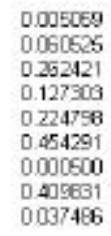 & 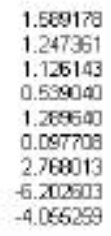 & $\begin{array}{l}0.1133 \\
0.2135 \\
0.2512 \\
0.5904 \\
0.1984 \\
0.9222 \\
0.00061 \\
00000 \\
0.0001\end{array}$ & \\
\hline 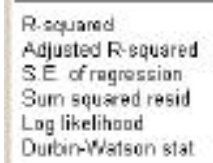 & 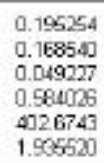 & \multicolumn{2}{|c|}{ 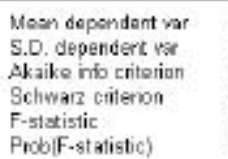 } & $\begin{array}{r}0.0149848 \\
0.053987 \\
.3 .149394 \\
-3.026222 \\
7.390158 \\
0.000000\end{array}$ & $\begin{array}{l}\text { nipersthy } \\
\text { guss sohom }\end{array}$ \\
\hline EVIEWS Tutorial 1 & & & & & (-) Roy Batchelor 2000 \\
\hline
\end{tabular}


Specific model:stage two

\begin{tabular}{|c|c|c|c|c|}
\hline \multicolumn{4}{|c|}{ Equation UNTITLED Workfile: FT500M } & \multirow{2}{*}{ scids! } \\
\hline ViexulFices 0tiocts Firk & Namel Froszed & Estmale For & casil Stats $/ R$ & \\
\hline \multicolumn{5}{|c|}{$\begin{array}{l}\text { Dapendent Yariable: DLFT50 } \\
\text { Method: Least Squares } \\
\text { Date: 11,0200 Time: 11:14 } \\
\text { Sample(adjusted): 1975:02 1966.12 } \\
\text { Included obsarrations: } 251 \text { after adjusting endpoints. }\end{array}$} \\
\hline Wariable & Cosficiant & Std. Enor & t-Statistic & Prob. \\
\hline $\begin{array}{c}\mathrm{C} \\
\text { DCONF } \\
\text { RES(-1) }\end{array}$ & $\begin{array}{r}0.015635 \\
0.01246 \\
0.128131\end{array}$ & $\begin{array}{l}0.003412 \\
0.000523 \\
0.039385\end{array}$ & $\begin{array}{l}4592059 \\
2.391235 \\
3.330063\end{array}$ & $\begin{array}{l}0.0000 \\
0.0180 \\
0.0010\end{array}$ \\
\hline $\begin{array}{l}\text { R-squared } \\
\text { Adjusted R-squared } \\
\text { S.E. of regression } \\
\text { Sum squared resid } \\
\text { Log likelihood } \\
\text { Durbin-Watsun stat }\end{array}$ & $\begin{array}{l}0.053258 \\
0.045623 \\
0.054048 \\
0.724444 \\
377.7459 \\
1.005954\end{array}$ & \multicolumn{2}{|c|}{$\begin{array}{l}\text { Mean dependent var } \\
\text { S.D. dependent var } \\
\text { Akaike info criterion } \\
\text { Schukarz criterion } \\
\text { F-statistic } \\
\text { Probif-statistic] }\end{array}$} & $\begin{array}{r}0.015741 \\
0.055324 \\
-2.905023 \\
-2.943856 \\
6.975130 \\
0.001129\end{array}$ \\
\hline
\end{tabular}

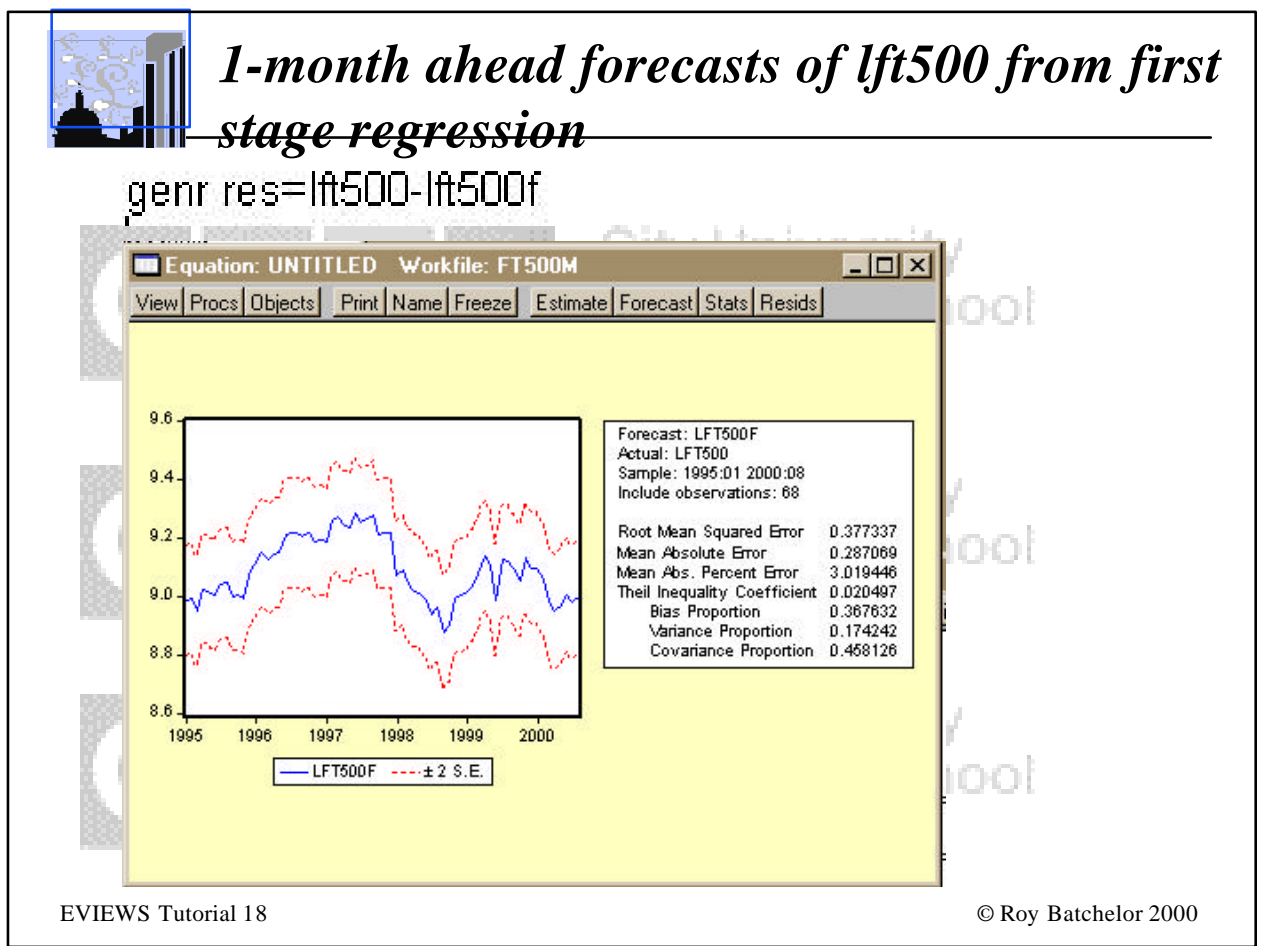



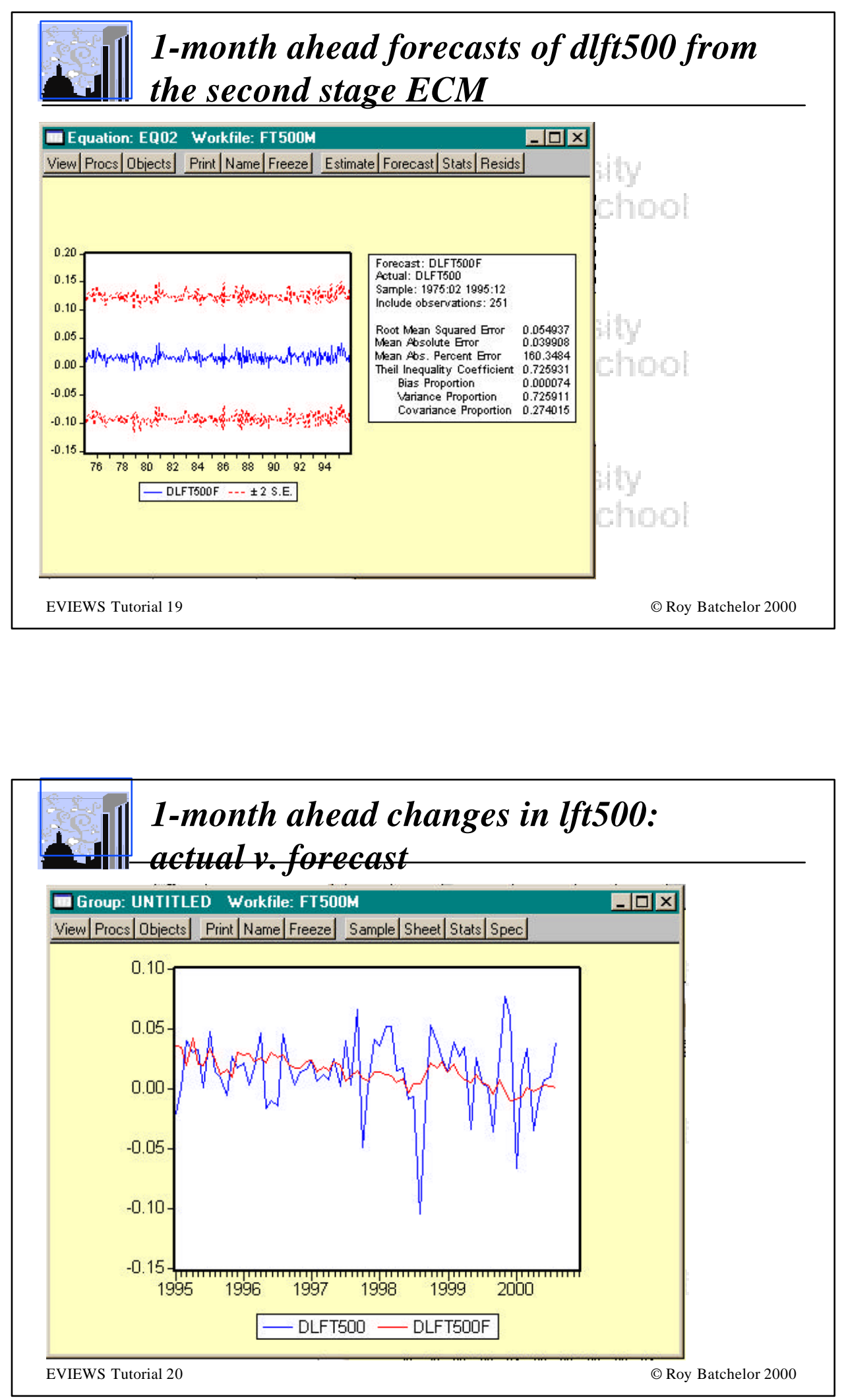


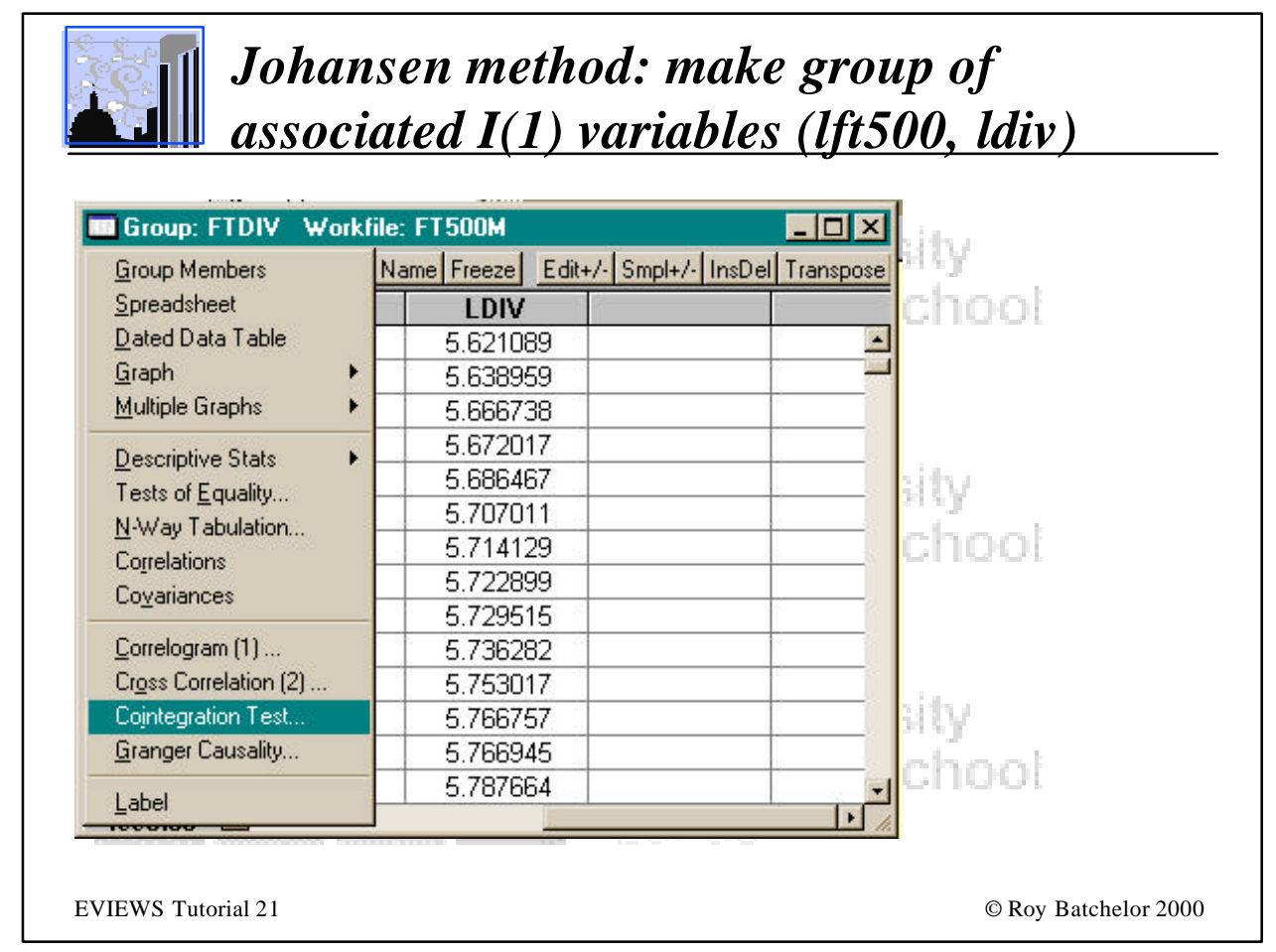

Set up Johansen procedure

\begin{tabular}{|c|c|}
\hline Johansen Cointegration Test & $\underline{x}$ \\
\hline Cointegrating Equation (CE) and VAR specification: & \multirow[b]{2}{*}{$\begin{array}{l}\text { Information: } \\
\text { The test VAR is } \\
\text { estimated in } \\
\text { differenced form. } \\
\text { CE and data trend } \\
\text { assumptions apply to } \\
\text { levels. } \\
\text { xxxxx Warning } \times x \times x \times \\
\text { Test critical values } \\
\text { were derived } \\
\text { assuming no } \\
\text { exogenous series. }\end{array}$} \\
\hline $\begin{array}{l}\text { Test assumes no deterministic trend in data: } \\
\text { No intercept or trend in CE or test VAR } \\
\text { Intercept (no trend) in CE - no intercept in VAR } \\
\text { Test allows for linear deterministic trend in data: } \\
\text { Intercept (no trend) in CE and test VAR } \\
\text { Intercept and trend in CE - no trend in VAR } \\
\text { Test allows for quadratic deterministic trend in data: } \\
\text { Intercept and trend in CE - linear tend in VAR } \\
\text { Summary: } \\
\text { Summarize all } 5 \text { sets of assumptions }\end{array}$ & \\
\hline $\begin{array}{l}\text { Exogenous series in VAR: } \\
\text { (don't include } C \text { or trend) }\end{array}$ & \\
\hline Lag intervals (pairs) in VAR: 11 & SCancel \\
\hline
\end{tabular}




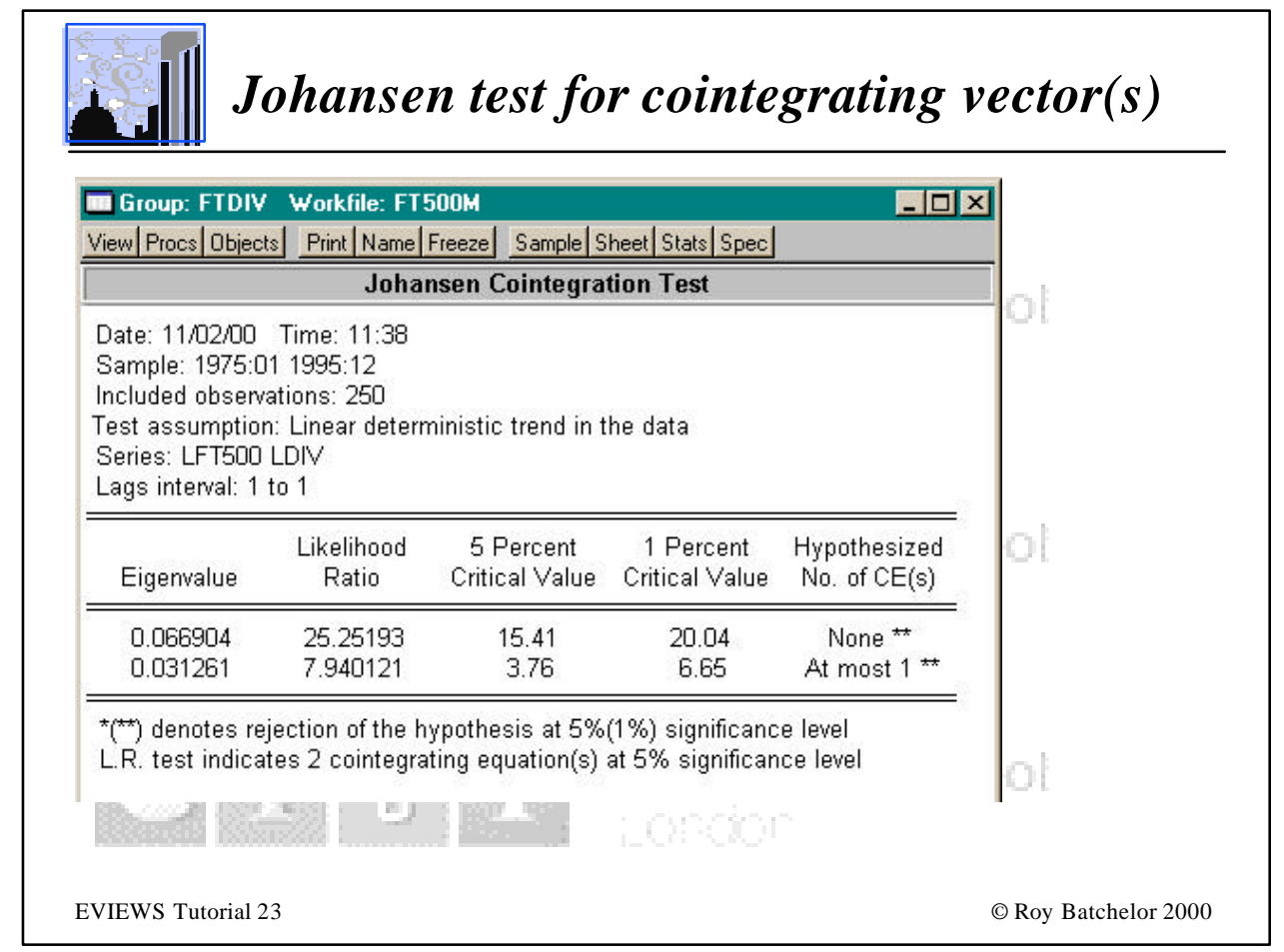

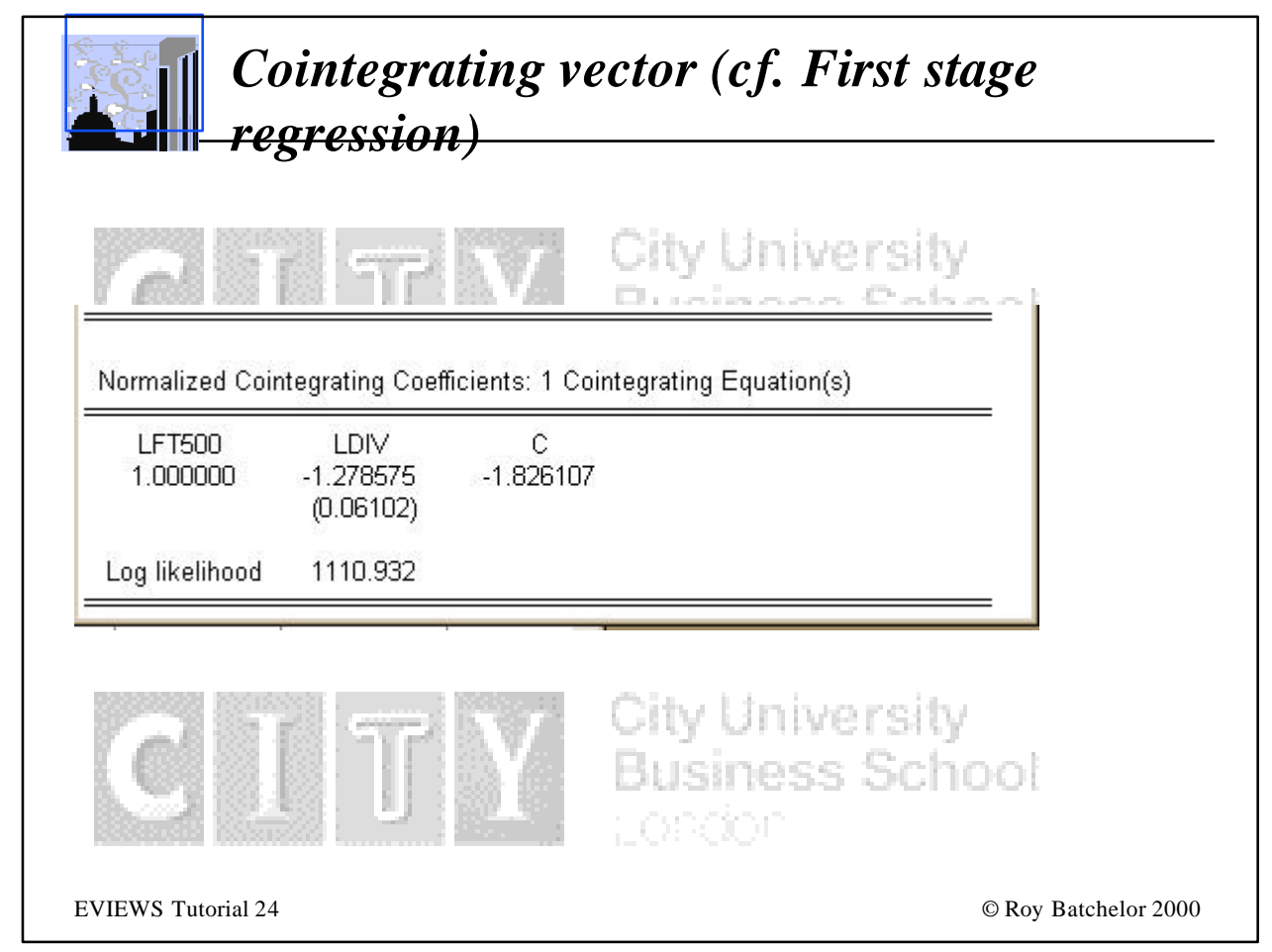


Set up VAR-ECM
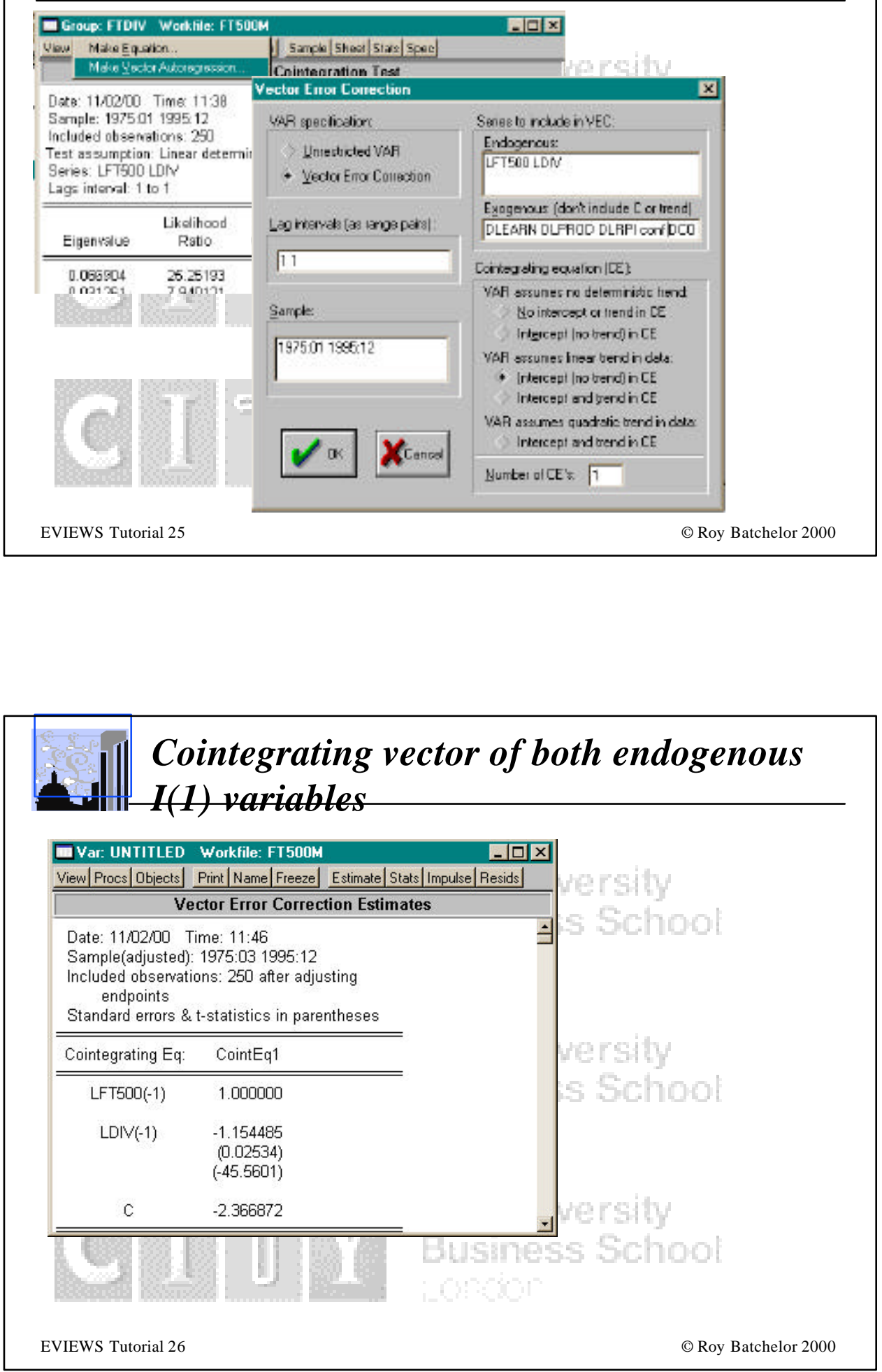


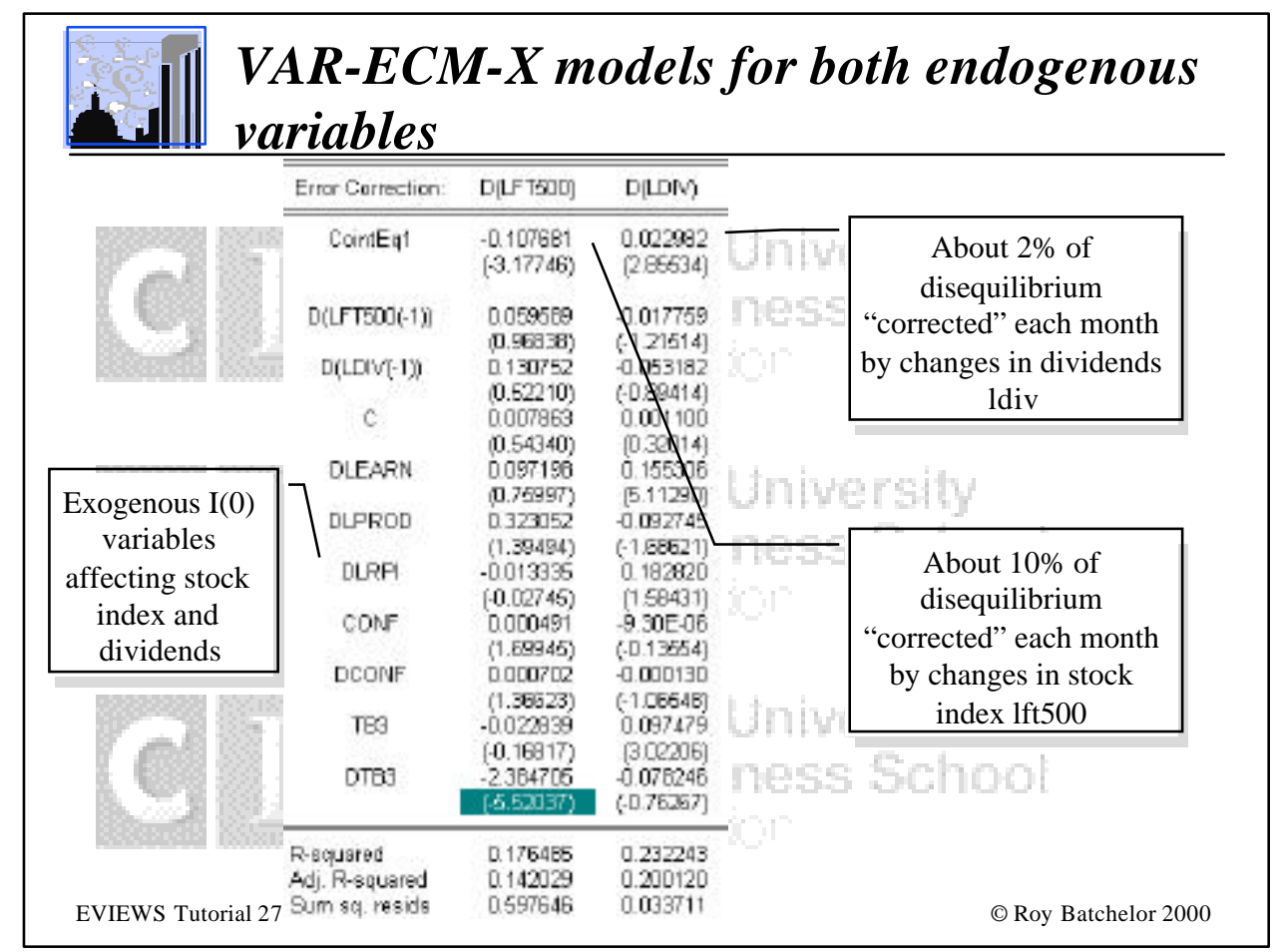

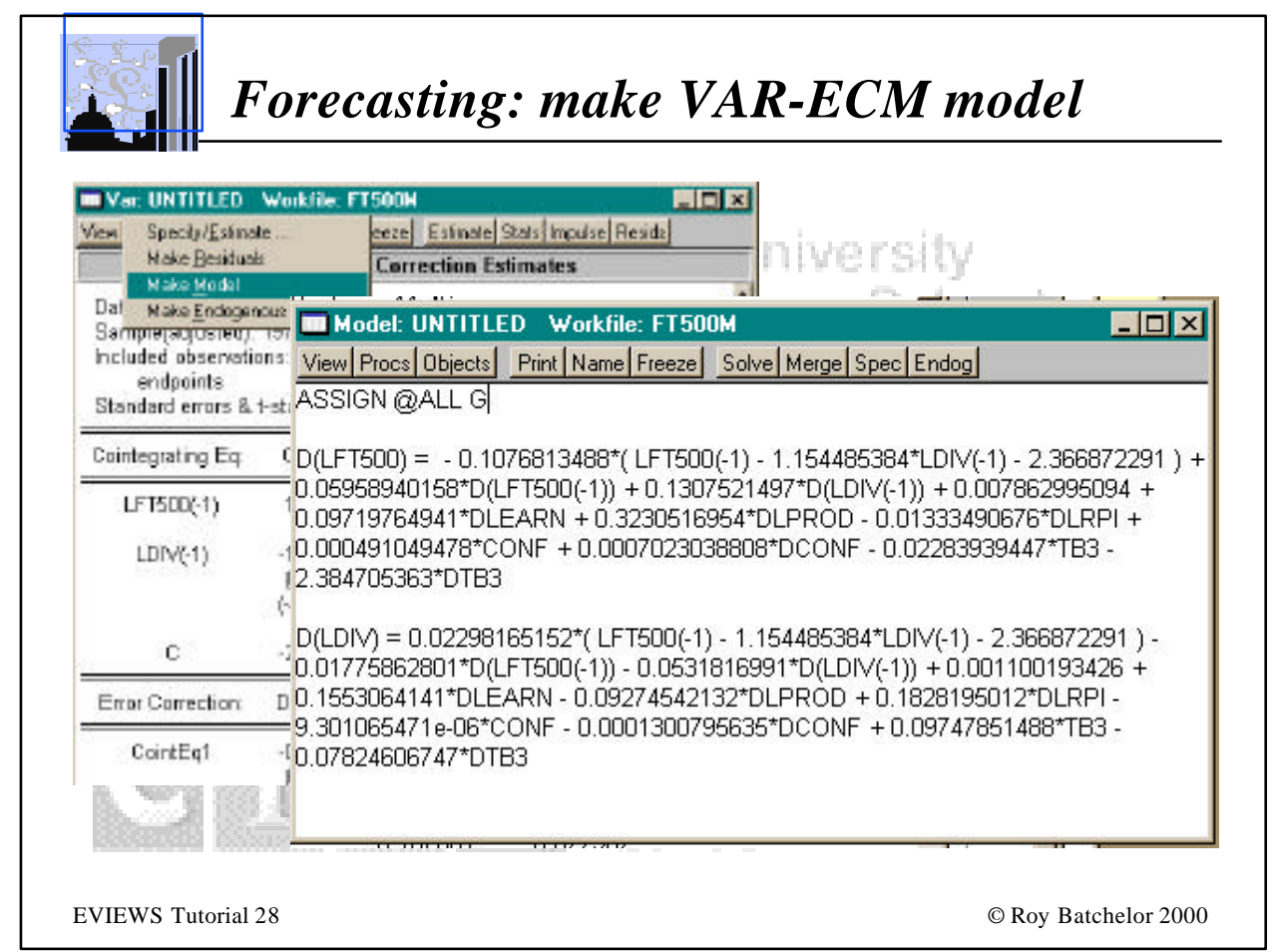



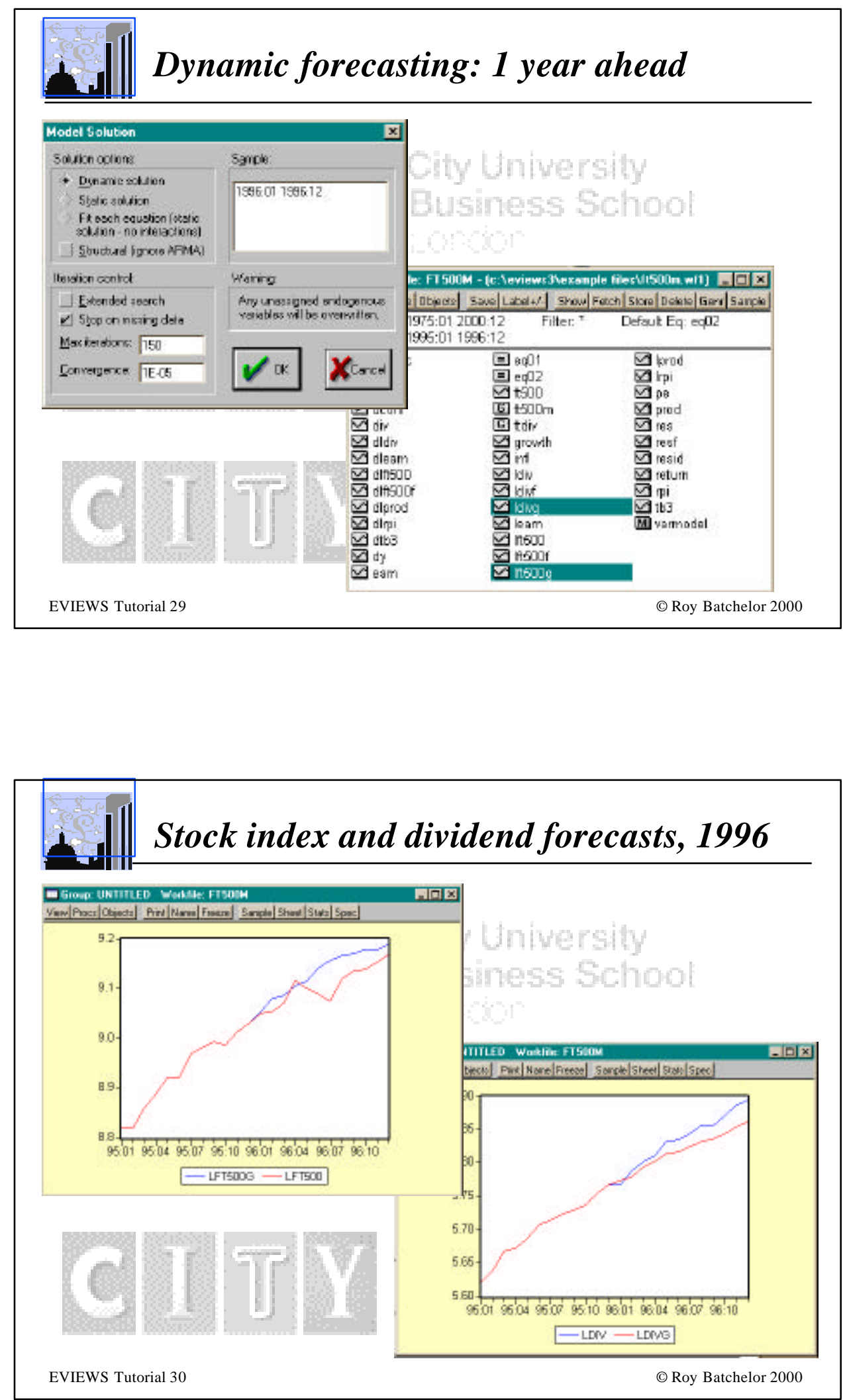


\section{Updated model (1975-98)}

\begin{tabular}{|c|c|}
\hline \multicolumn{2}{|l|}{ Wodel UNTITLED Wonkfile: FT $500 \mathrm{M}$} \\
\hline 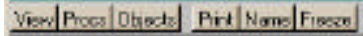 & Sotho/Mans/5pec/Endod \\
\hline \multirow{2}{*}{\multicolumn{2}{|c|}{ 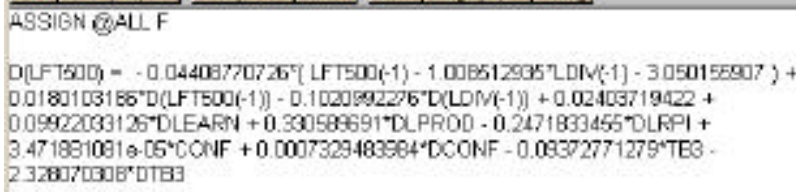 }} \\
\hline & \\
\hline \multicolumn{2}{|c|}{ 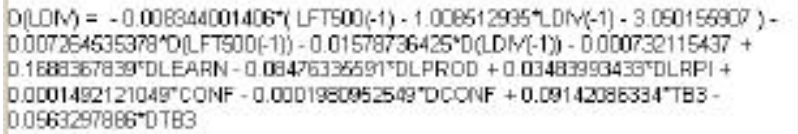 } \\
\hline
\end{tabular}
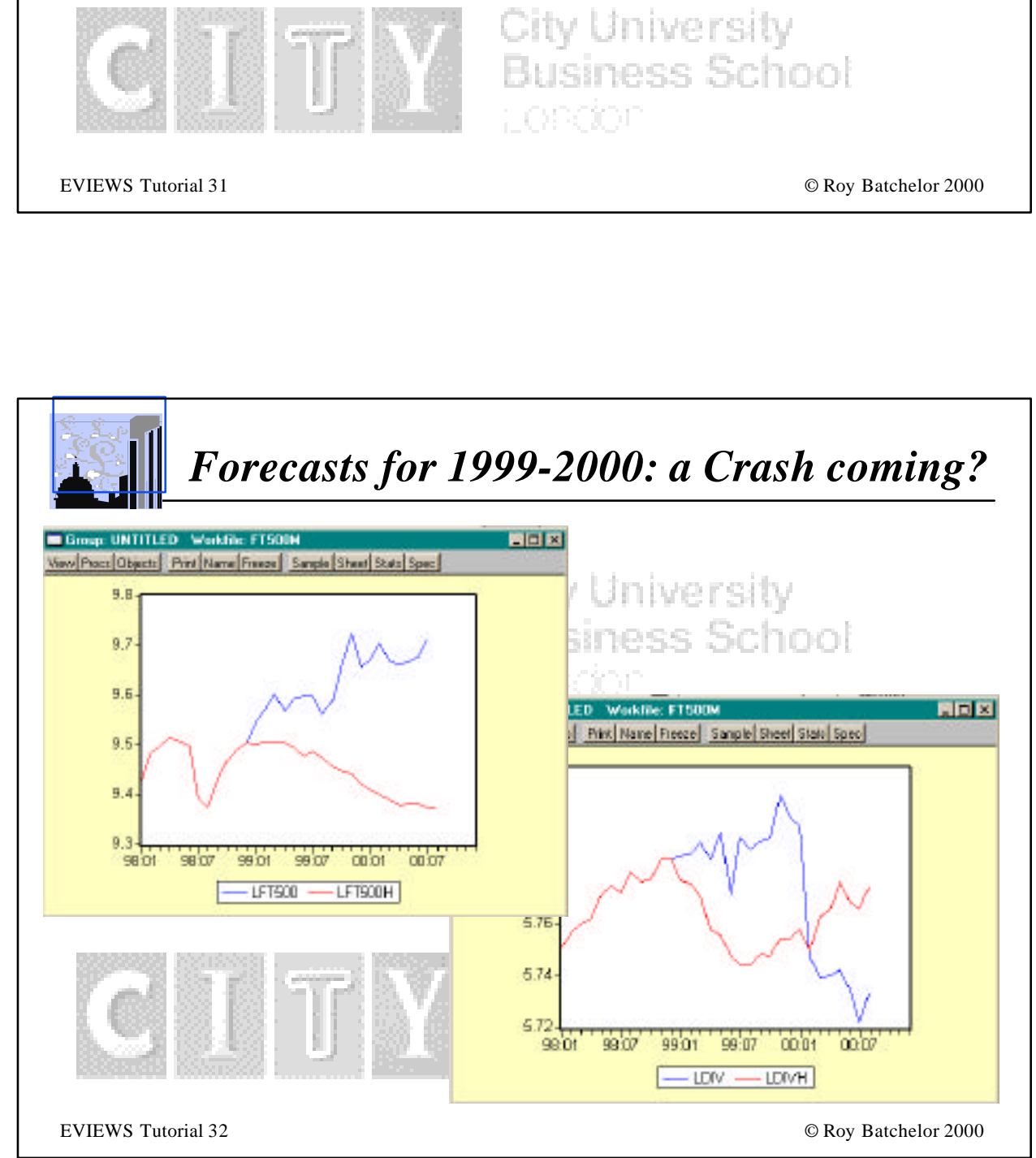